\section{In the news}

\section{OF COWS AND MEN}

Researchers in the UK have requested permission to generate embryos by inserting human nuclei into cow eggs that have been depleted of their own nuclei. These embryos, which would not be allowed to develop for longer than 14 days, would be used for stem-cell research aimed at treating neurological disorders such as Alzheimer's and Parkinson's disease. Another team of researchers wants to use a similar technique to study how eggs can reprogramme adult cells into stem cells (BBC News, 7 Nov 2006).

"We are concerned that the current state of the technology means that hundreds of eggs from young woman will be required to generate a single human embryonic stem cell line. Therefore, we consider it more appropriate to use nonhuman eggs from livestock as a surrogate", says Stephen Minger, one of the researchers (Telegraph, 7 Nov 2006).

Although not an entirely new technique, mixing human and animal eggs and sperm is prohibited in the UK by the Human Fertilisation and Embryology Act 1990. But, the applicants are hopeful that this could change within a few months, opening up a new range of research possibilities.

Unsurprisingly, some members of the public are critical. Calum MacKellar of the Scottish Council on Human Bioethics is concerned that these studies "might also undermine human dignity and human rights" (BBC News, 7 Nov 2006).

However, Evan Harris, a member of parliament and a member of the Commons Science and Technology Select Committee, raises an opposing point: "If human benefits can be derived by perfecting therapeutic cloning techniques or from research into subsequentlyderived stem cells, then it would actually be immoral to prevent it just because of a 'yuck' factor" (BBC News, 7 Nov 2006).

Asher Mullard

DNA DAMAGE

\title{
The road of death
}

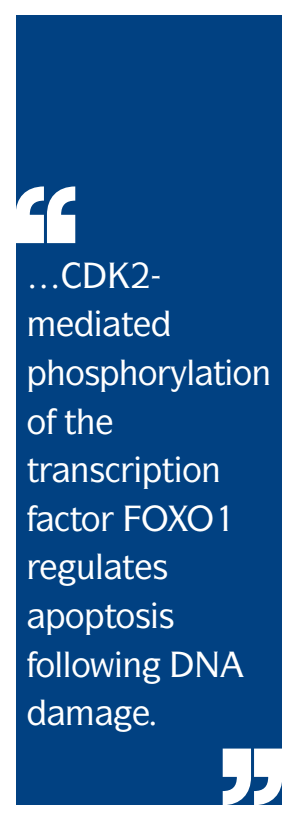

DNA damage activates checkpoint pathways that induce cell-cycle arrest and subsequent DNA repair or cell death. Cyclin-dependent kinase-2 (CDK2) is inhibited by DNA damage, but whether CDK2 has a role in DNA-damage-induced cell death has been unknown. Donald Tindall and colleagues have addressed this question and found that CDK2-mediated phosphorylation of the transcription factor FOXO1 regulates apoptosis following DNA damage.

Given that FOXO transcription factors control a number of celldeath genes, Tindall and co-workers investigated a possible connection between CDK2 and the activity of FOXO proteins. They found that endogenous CDK2 phosphorylates FOXO1 at residue Ser249, and to a lesser degree residue Ser298, in vitro. This effect was abolished by a CDK inhibitor. The authors developed a phosphorylation-specific antibody and provided in vivo evidence for the CDK2-dependent phosphorylation of FOXO1. The antibody recognized wild-type FOXO1, but not a Ser249 to Ala249 (S249A) mutant of FOXO1. Small-interfering RNA (siRNA)mediated silencing of CDK2 led to a decrease in FOXO1 phosphorylation, whereas FOXO1 phosphorylation was increased in cells that had been transfected with a constitutively active CDK2 mutant.

The transcriptional activity of FOXO1 was decreased in the presence of ectopically expressed CDK2 and its regulator cyclin E. By contrast, its transcriptional activity was increased

\section{METASTASIS}

\section{Contract and move}

The regulated assembly and disassembly of cell-extracellular matrix junctions (focal adhesions) contributes to cell motility and tumour invasion. Rho-ROCK signalling promotes focal adhesion disassembly at the rear of the cell by inducing contractile forces that are dependent on the phosphorylation of myosin light chain-2 (MLC2). Isacke and colleagues now show that the Rho-ROCK-MLC2 signalling pathway is activated to generate contractile forces at distinct subcellular locations through endosomal trafficking.

Using human cancer cell lines the authors compared the small interfering RNA (siRNA)-mediated depletion of the promigratory transmembrane receptor
ENDO180 (also known as CD280) and two other endocytic receptors. ENDO180 is constitutively recruited into clathrin-coated pits on the cell surface, which are then internalized into intracellular endosomes. Only the depletion of ENDO180 induced tail-retraction defects, indicative of

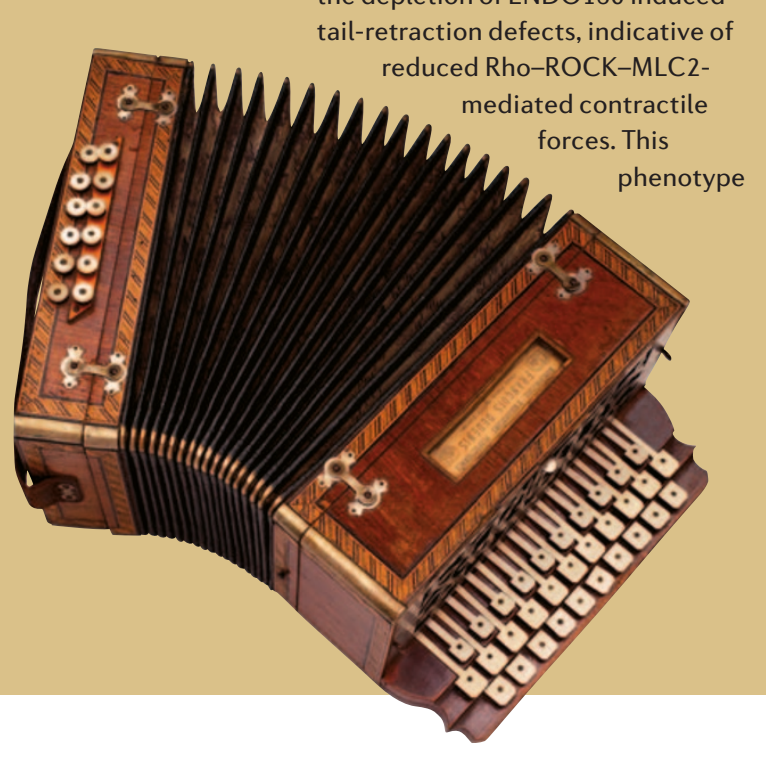


by co-transfection of the tumour suppressor phosphatase and tensin homologue (PTEN), which promotes a decrease in FOXO1 phosphorylation. The increased transcriptional activity of FOXO1 was abolished in the presence of wild-type CDK2, but not by a kinase-inactive CDK2 mutant. Also, the inhibitory effect of CDK2 was diminished in a FOXO1 (Ser249, Ser298) double mutant. Together, these data indicate that the transcriptional activity of FOXO1 is repressed primarily by the CDK2mediated phosphorylation of Ser249.

As residue Ser 249 of FOXO1 is located adjacent to a nuclear localization motif, and as overexpression of CDK2 causes the cytoplasmic localization of wild-type FOXO1 but not of the phosphorylation-resistant S249A mutant, CDK2 phosphorylation is thought to induce the cytoplasmic localization of FOXO1 from the nucleus and thereby repress its transcriptional activity.

When Tindall and colleagues treated cells with a DNA-damaging agent, camptothecin or $\gamma$-irradiation, phosphorylation of FOXO1 at Ser249 was abolished. siRNA-mediated silencing of either the CHK1 or CHK2 kinase of the DNA-damage-mediated checkpoint pathway partially blocked the camptothecin-induced decrease in FOXO1 phosphorylation. These data indicate that DNA-damaging agents regulate FOXO1 by controlling CHK1- and CHK2-dependent checkpoint pathways. FOXO1-mediated cell death in response to DNA damage was observed both in p53-deficient and p53-containing cell lines, which implies that FOXO1 contributes to the apoptotic response to DNA damage independently of p53.

In summary, CDK2-mediated regulation of FOXO1 represents a novel pathway that links DNA damage to apoptosis. Interestingly, in cells in which FOXO1 was silenced, overexpression of FOXO3a or FOXO4 restored DNA-damage-induced apoptosis. It is therefore important to examine the roles of other FOXO proteins in the selective killing of cells in response to DNA damage.

\section{Arianne Heinrichs}

ORIGINAL RESEARCH PAPER Huang, H. et al. CDK2-dependent phosphorylation of FOXO1 as an apoptotic response to DNA damage. Science 314, 294-297 (2006)

FURTHER READING Bartek, J. et al. Checking on

DNA damage in $\mathrm{S}$ phase. Nature Rev. Mol. Cell Biol. 5, 792-804 (2006)

WEB SITE

Donald Tindall's laboratory:

http://mayoresearch.mayo.edu/mayo/research/ staff/tindall_dj.cfm was comparable to that observed when cells were treated with Rho or ROCK inhibitors, establishing ENDO180-RhoROCK-MLC2 as a functional pathway. Moreover, ENDO180 siRNA or a ROCK inhibitor reduced the phosphorylation of three other ROCK substrates, LIM kinase- 1 and -2 , and myosin phosphatase-1.

Isacke and colleagues also show that ENDO180 is required for the spatial activation of Rho-ROCK-MLC2. Using cell lines that stably express an ENDO180 mutant that cannot be internalized, the authors demonstrate that ROCK cannot be recruited to focal adhesions. This shows that intracellular ENDO180-containing endosomes are required to activate $\mathrm{Rho-ROCK}$.

ENDO180 is highly expressed in motile cells, particularly fibroblasts, and has an established role in collagen internalization. Bugge and colleagues have shown that mice undergoing polyomavirus-induced mammary carcinogenesis that also have a targeted deletion in ENDO180 have impaired tumour expansion, and therefore a reduced tumour burden. Isacke and colleagues suggest that this reduction in tumour burden could arise both from the inability of ENDO180-null fibroblasts to remodel the extracellular matrix and from impaired motility in the tumour. Indeed, targeting ENDO180 might benefit anticancer therapy by causing the retention of a tumour-inhibitory matrix.

\section{Gemma Alderton, Associate Editor,} Nature Reviews Cancer

ORIGINAL RESEARCH PAPER Sturge, J. et al. Endosomes generate localized Rho-ROCKMLC2-based contractile signals via Endo 180 to promote adhesion disassembly. J. Cell Biol. 175, 337-347 (2006)

FURTHER READING Curino, A. C. et al. Intracellular collagen degradation mediated by uPARAP/Endo 180 is a major pathway of extracellular matrix turnover during malignancy. J. Cell Biol. 169, 977-985 (2005)

\section{IN BRIEF}

\section{$\Rightarrow$ APOPTOSIS}

Histone $\mathrm{H} 2 \mathrm{~B}$ deacetylation at lysine 11 is required for yeast apoptosis induced by phosphorylation of $\mathrm{H} 2 \mathrm{~B}$ at serine 10 .

Ahn, S.-H. et al. Mol. Cell 24, 211-220 (2006)

Histone modifications are known to regulate a number of cellular processes. In growing yeast, the histone protein $\mathrm{H} 2 \mathrm{~B}$ is acetylated on Lys11, whereas phosphorylation of the adjacent Ser10 residue has been linked to the induction of apoptosis. Ahn et al. now show that hydrogen peroxide treatment of growing cells first causes Lys11 to be deacetylated, followed by the subsequent phosphorylation of Ser10 and apoptosis. The deacetylase enzyme Hos3 mediates the first step, whereas phosphorylation depends on the kinase Ste20. The authors propose that a concerted series of histone modifications control the switch from cell growth to cell death.

\section{$\Rightarrow$ DEVELOPMENT}

Canonical notch signaling functions as a commitment switch in the epidermal lineage.

Blanpain, C. et al. Genes Dev. 20, 3022-3035 (2006)

The formation of mammalian epidermis involves the differentiation and migration of basal-layer progenitor cells to suprabasal spinous cells. Blanpain and colleagues show that Notch signalling commits dividing progenitor cells to becoming spinous cells. Activated Notch intracellular domain (NICD) and its signalling partner RBP-J are both required to repress expression of progenitor-cell genes and to activate spinous-cell gene expression. Spinous-cell gene activation depends on the NICD-RBP-J target gene HES1, whereas progenitor-cell gene repression occurs through a HES1-independent mechanism.

\section{$\Rightarrow$ SIGNAL TRANSDUCTION}

Global, in vivo, and site-specific phosphorylation dynamics in signaling networks.

Olsen, J. V. et al. Cell 127, 635-648 (2006)

Protein phosphorylation functions as a reversible switch in many signalling cascades. Using a high-throughput massspectrometry-based technique, the authors detected 6,600 protein phosphorylation sites in HeLa cells and monitored changes in phosphorylation during epidermal growth factor stimulation. Phosphorylation is modulated by at least twofold at $14 \%$ of phosphorylation sites and proteins that contain multiple phosphorylation sites are often regulated independently.

\section{$\Rightarrow$ SUMOYLATION}

Regulation of MBD1-mediated transcriptional repression by SUMO and PIAS proteins.

Lyst, M. J. et al. EMBO J. 26 Oct 2006 (doi:10.1038/sj.emboj.7601404)

Methylated DNA can serve as a binding site for methyl$\mathrm{CpG}$-binding domain (MBD) proteins, which, in turn, recruit co-repressor complexes that modify chromatin into an inactive state. Lyst et al. found that the activity of MBD1 can be regulated by sumoylation near its $C$ terminus by two PIASfamily members, PIAS1 and PIAS3. Sumoylated MBD1 still binds to methylated DNA, but neither recruits the histone methylase SETDB1 nor silences gene expression in HeLa cells. MBD1 is proposed to function as a scaffolding platform on methylated DNA that can be regulated through sumoylation. 